\title{
Uso de medicamentos potencialmente inapropriados para idosos em uma instituição de longa permanência
}

\author{
Luana Pereira da Rosa*, Silvia Dal Bó
}

Universidade do Extremo Sul Catarinense-UNESC, Criciúma, SC, Brasil

\section{Histórico do Artigo \\ Recebido em: \\ 24/04/2021 \\ Aceito em: \\ 08/09/2021}

\section{Palavras-chave:}

Idosos; polimedicação; Instituição de longa permanência; interações de medicamentos

Keywords:

Elderly; polypharmacy; long-stay institution; drug interactions

\begin{abstract}
RESUMO
Vivemos uma mudança no perfil demográfico, o decrescimento da média de filhos por mulher, em contraponto com o aumento da expectativa de vida. Em um futuro próximo, uma grande parcela da população será composta por idosos. Este fato acarreta um problema de saúde pública, necessitando de um olhar cauteloso em relação a proteção e promoção da saúde do idoso. Sabese que com o passar dos anos a população tende a apresentar patologias características da idade, resultando em pacientes polimedicados e/ou fazendo uso de medicamentos inapropriados. $\mathrm{O}$ objetivo dessa pesquisa foi avaliar as prescrições de 35 pacientes idosos residentes em uma instituição de longa permanência no Extremo Sul Catarinense, identificando o uso de medicamentos inapropriados conforme Critério de Beers e Start/Stop, além de pontuar possíveis interações medicamentosas. Com base nos dados obtidos, pode-se afirmar que $60 \%$ dos indivíduos são do sexo feminino, a maioria com idade entre 66 e 85 anos; 6\% são portadores de polipatologias e $66 \%$ em uso de 5 a 10 medicamentos. Dos medicamentos utilizados $30 \%$ são inapropriados para idosos e 216 interações medicamentosas foram encontradas, destas, $91 \%$ tratam-se de interações moderadas e $9 \%$ de interações graves. A pesquisa possibilitou chamar a atenção dos profissionais de saúde demonstrando números elevados em relação a interações e uso de medicamentos impróprios, além de relatar o impacto dessa realidade e seus agravos na qualidade de vida do idoso.
\end{abstract}

Use of potentially inappropriate drugs for the elderly in an institution for long stay

\section{ABSTRACT}

We live in a change in the demographic profile, the decline of the average number of children per woman, in contrast with the increase in life expectancy. In the near future, a large portion of the population will be composed by the elderly. This fact leads to a public health problem, requiring a careful look in relation to protecting and promoting the health of the elderly. It is known that with the passing of years the population tends to present pathologies characteristics of age, resulting in patients polymedicated and/or making use of inappropriate medications. The objective of this research was to assess the requirements of 35 elderly patients residing in an institution of long permanence in South Catarinense Extreme, identifying the use of inappropriate medications as criterion of beers and Start/Stop, as well as score possible drug interactions. Based on the data obtained, it can be argued that $60 \%$ of individuals are female, most aged between 66 to 85 years; $6 \%$ are bearers of polipatologias and $66 \%$ in use from 5 to 10 medicines. The medications used $30 \%$ are inappropriate for elderly and 216 drug interactions were found, of these, $91 \%$ are moderate interactions and $9 \%$ of serious interactions. The survey enabled us to draw the attention of health professionals demonstrating high numbers in relation to the interactions and inappropriate use of medications, in addition to reporting the impact of this reality and their harm in the quality of life of the elderly.

\section{Introdução}

De acordo com o Estatuto do Idoso (1), no Brasil, considera-se idoso todo cidadão com idade igual ou acima de 60 (sessenta) anos. O estatuto ainda traz que é ofício da família, da sociedade, da comunidade e do Poder Público garantir ao idoso o direito à vida, à saúde, à alimentação, à educação, à cultura, ao esporte, ao lazer, ao trabalho, à cidadania,

\footnotetext{
* Autor correspondente: luanadarosa201@gmail.com (da Rosa L.P.)
} 
à liberdade, à dignidade, ao respeito e à convivência familiar e comunitária. Com o passar das décadas no Brasil, o envelhecimento populacional teve um aumento acentuado, estando na quarta posição entre os 35 países mais populosos do mundo, atrás apenas da República da Coreia, Tailândia e Japão. Em contrapartida, a taxa de fecundidade brasileira caiu de 6,16 filhos por mulher para 1,57 filhos entre 1940 e 2014 (2).

Com a mudança gerada no perfil demográfico devido ao decrescimento do número médio de filhos e a queda das taxas de mortalidade, acredita-se que no final do século XXI, 4 em 10 brasileiros terá 60 anos ou mais (3).

A consequência da mudança do perfil demográfico associado às alterações características do envelhecimento modifica o perfil epidemiológico do Brasil para uma hegemonia de doenças crônicas prevalentes dessa população, como por exemplo, doenças cardiovasculares, hipertensão, derrame, diabetes, câncer, doença pulmonar obstrutiva crônica, doenças musculoesqueléticas, doenças mentais (principalmente demência e depressão), cegueira e diminuição da visão. Em paralelo, observa-se um aumento da utilização de medicamentos para prevenir, controlar ou alcançar a cura, diminuindo as limitações no desenvolvimento das atividades no cotidiano e aumentando o prognóstico de vida com um possível retardo da evolução patológica (4).

Frente a essa alteração do perfil epidemiológico, a indústria farmacêutica passa a observar o paciente idoso como uma fonte de lucro, disponibilizando uma gama gigantesca de opções farmacológicas inseridas em um contexto de mercado capitalista. Por outro ângulo, o medicamento desperta ao paciente uma certa expectativa em relação ao tratamento, mas nem sempre irá apresentar a segurança e a efetividade necessárias para a condição clínica denotada. Além do mais, todo o medicamento pronuncia-se de uma forma bivalente, podendo conduzir a um efeito benéfico ou prejudicial à saúde, dependendo da forma de uso, dose e tempo de tratamento (5).

As complicações relacionadas à farmacoterapia tem sido uma verdadeira epidemia moderna e estão produzindo por muitas vezes mais danos ao paciente do que as próprias doenças que originaram a inevitabilidade da utilização desses medicamentos, resultando em idosos polimedicados e internações hospitalares de alto custo que poderiam ser evitadas (6). As prescrições farmacológicas tratam-se do principal tipo de intervenção terapêutica, e devido a esse fato, garantir a escolha do medicamento ideal para cada patologia e para cada paciente, torna-se um processo de grande responsabilidade, um ato complexo e muito vulnerável à iatrogenia, principalmente quando se faz referência a saúde do idoso (7).

Uma prescrição inapropriada traz o risco de reações adversas que deveriam ser evitadas quando há evidência de medicamentos de alternativa igual ou com maior efetividade e menor risco para tratar a mesma condição clínica, resultando na diminuição da frequência/tempo de uso medicamentoso e a prática de uso exacerbado de múltiplos medicamentos causadores de interações. A adequação dessas prescrições tem sido fundamentada em instrumentos com o propósito de melhorar a qualidade e segurança das prescrições para idosos, os mais citados e utilizados são os Critérios de Beers dos Estados Unidos e a Ferramenta de Triagem de Prescrições Potencialmente Inapropriadas para Idosos (STOPP) da Irlanda. Pioneiro em instituições de longa permanência, o Critério de Beers possui atualização mais recente em 2019, incluindo medicamentos e grupos farmacológicos que devem ser evitados em todos os idosos, os que devem ser evitados em determinada condição clínica e, por último, aqueles que devem ser utilizados com precaução (8).

Dessemelhante às demais atuações do farmacêutico, a prática de serviços clínicos deve ser centralizada no cuidado ao paciente e não no medicamento, apesar das divergências relacionadas à farmacoterapia ainda serem o ponto chave para a resolução do problema. 
As percepções e condutas referentes ao uso do medicamento variam de pessoa para pessoa através da cultura, experiências adquiridas, instruções em saúde pré-estabelecidas e da visão da doença e tratamento. É papel do farmacêutico compreender essa singularidade, visando sempre o bem-estar global do paciente, facilitando sempre o processo de aceitação e tratamento, avaliando a necessidade medicamentosa, melhorando a adesão, o uso, a efetividade e a segurança da farmacoterapia (5).

Tendo em vista o assunto abordado, o presente estudo traz a avaliação do número de prescrições de medicamentos inapropriados associados a uma alta taxa de efeitos adversos, seguidos da avaliação de possíveis interações medicamentosas em uma instituição de longa permanência do Extremo Sul Catarinense.

\section{Metodologia}

Esse estudo tratou-se de uma pesquisa descritiva observacional referente a utilização de medicamentos inapropriados para idosos em uma instituição de longa permanência de caráter filantrópico do Extremo Sul Catarinense, a mesma conta atualmente com 35 moradores que possuem como suporte técnicas de enfermagem para cuidados e administração de medicamentos. Esse estudo abordou os medicamentos inapropriados utilizados por pacientes com idade igual ou superior a 60 anos, com critério de exclusão pacientes em cuidados paliativos, segundo o Critério de Beers publicado em 2019.

Após a submissão do presente pelo Comitê de Ética e Pesquisa em Seres Humanos (CEP) da Universidade do Extremo Sul Catarinense (UNESC), e aprovação sob o número de parecer 3.542.674, foram avaliados 35 prontuários de indivíduos residentes na instituição totalizando $100 \%$ da amostra. O período de coleta ocorreu entre 31/08/2019 e 07/09/2019.

Doravante, com a utilização de um formulário os dados dos participantes foram desmembrados por idade, sexo, número de medicamentos inapropriados prescritos conforme Critério de Beers e START/STOPP, número de medicamentos que devem ser evitados em determinadas condições clínicas/doenças, inapropriados que devem ser utilizados com cautela, bem como as classes farmacológica mais utilizadas de cada categoria. Além disso, foi caracterizado as interações medicamentosas de maior relevância clínica e seus possíveis efeitos adversos, através de ferramentas online como Uptodate, Drugs e Medscape, além da quantificação de participantes polimedicados e portadores de polipatologias.

A polimedicação, variável categórica, foi definida neste estudo como o uso concomitante de cinco ou mais medicamentos e a polipatologia foi definida como a presença de cinco ou mais doenças (9).

A pesquisa realizada tem como benefício a conscientização dos profissionais a respeito dos medicamentos potencialmente inapropriados para idosos e os riscos apresentados com o uso indevido dos mesmos, bem como, a apresentação dos dados obtidos para a instituição participante a fim de despertar um olhar mais criterioso e o uso racional de medicamentos, contribuindo com a qualidade e proteção da saúde do idoso.

Os dados foram armazenados em tabelas de Excel e gráficos conforme categoria de análise, com aplicação do formulário de dados na análise do prontuário conforme consta no apêndice. A metodologia de análise de dados baseou-se no conceito de test. $t$, utilizando conceitos estatísticos para rejeitar ou não a hipótese afirmada.

O termo de consentimento livre e esclarecido foi substituído pelo termo de confidencialidade, já que o responsável técnico pela instituição é o responsável legal pelos internos. Assim sendo, foi disponibilizado o termo de confidencialidade garantindo o sigilo de todos os dados obtidos na pesquisa. 


\section{Resultados}

Posteriormente a aprovação pelo Comitê de Ética e Pesquisa, compareceu-se a instituição a fim de realizar a coleta dos dados para análise, avaliando o número total de prontuários de pacientes residentes no local.

Em análise dos prontuários constatou-se que o sexo feminino prevalece na instituição em 60\% (21 indivíduos), em contrapartida com 40\% (14 indivíduos) do sexo masculino. Com relação a faixa etária encontrou-se indivíduos de 60 a 95 anos, com prevalência de 71 a 80 anos.

Seguindo para análise da saúde, constatou-se que apenas $6 \%$ dos pacientes são portadores de polipatologias, o qual foi definido anteriormente como a presença de cinco ou mais comorbidades, e $91 \%$ apresentavam de 1 a 4 comorbidades. Respectivamente na Figura 1 observa-se as principais patologias diagnosticadas, dentre elas predomina a Hipertensão Arterial Sistêmica que acomete 23\% dos pacientes, seguida da doença de Alzheimer que acomete $8 \%$ dos pacientes.

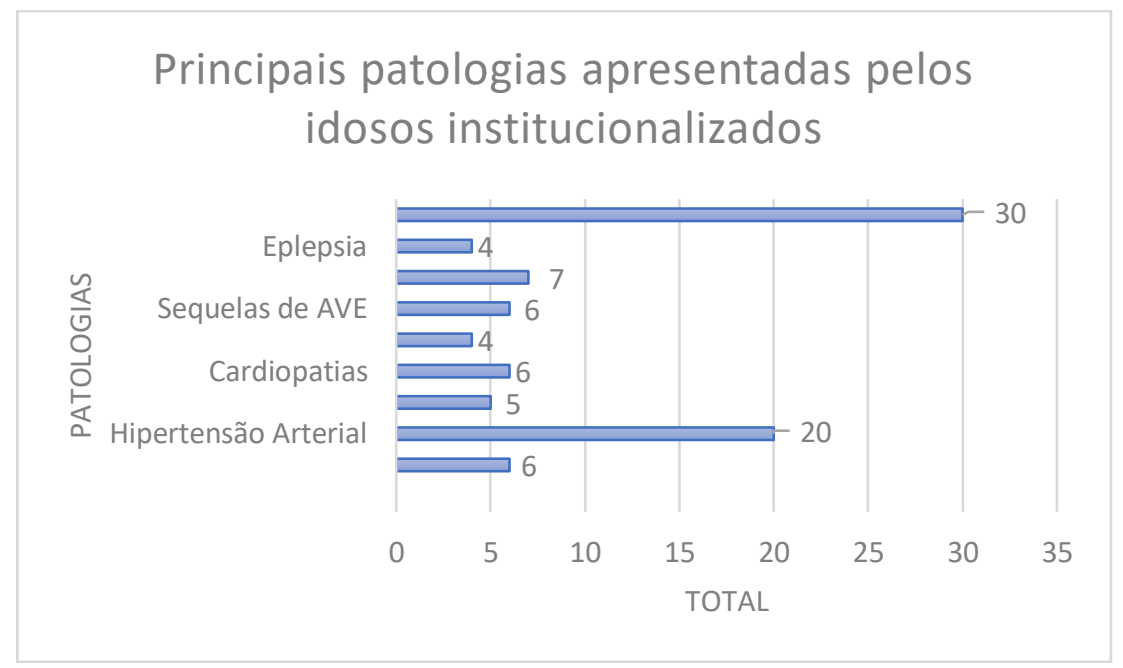

Figura 1 - Principais patologias apresentadas pelos idosos institucionalizados.

Subsequentemente desmembrou-se o número de medicamentos em quatro categorias: Uso de nenhum medicamento (3\%), 01 a 04 medicamentos (23\%), 05 a 10 medicamentos (66\%). Através do exposto demonstrou-se o total de $74 \%$ de pacientes sendo polimedicados, variável definida anteriormente como uso concomitante de cinco ou mais medicamentos.

Em avaliação farmacoterapêutica conforme o Critério de Beers e STOPP/START, observou-se um total de 217 medicamentos prescritos na instituição, sendo 30\% dos medicamentos utilizados são potencialmente inapropriados para idosos, presente estes na farmacoterapia de 30 pacientes, com predominância da classe terapêutica dos antipsicóticos atípicos, seguido dos benzodiazepínicos, inibidores da bomba de prótons e inibidores da recaptação de serotonina, conforme observa-se na Figura 2. 


\section{Medicamentos Inapropriados para idosos prescritos}

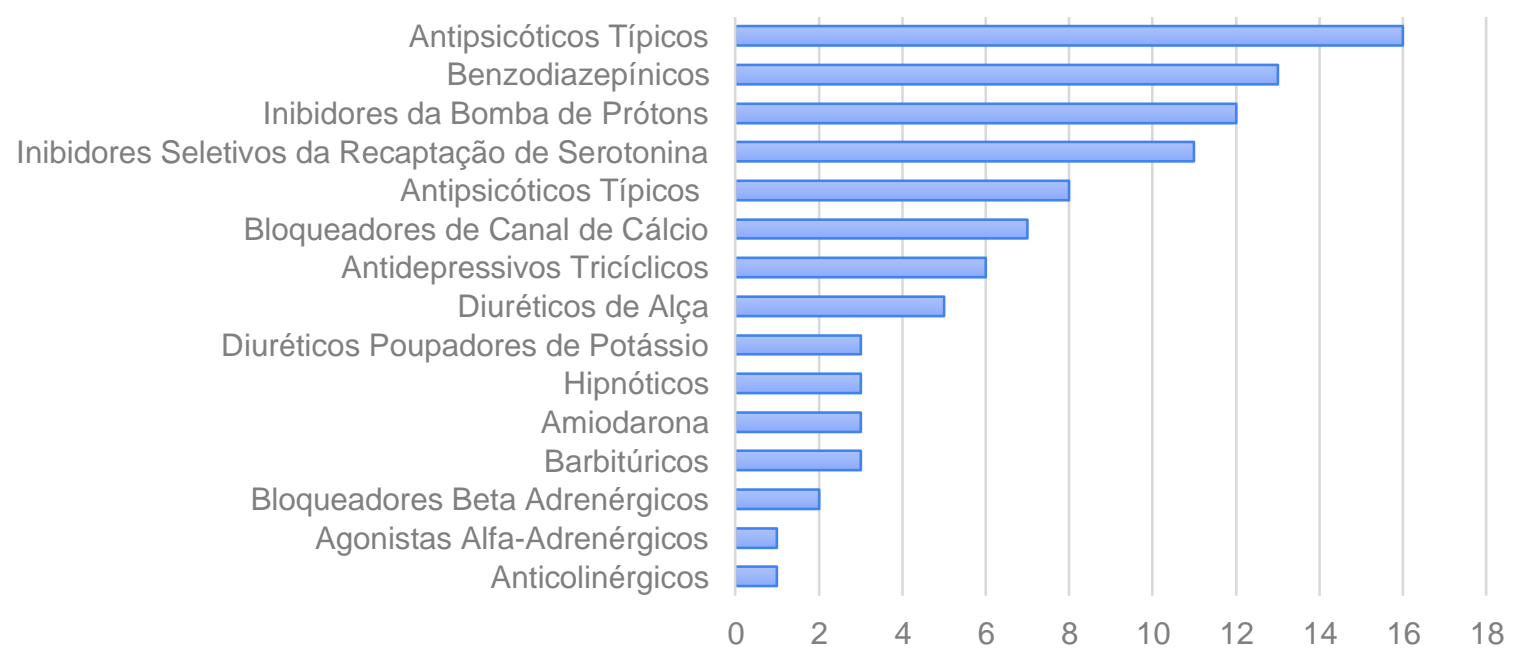

Figura 2 - Classes de medicamentos inapropriados para idosos prescritos na instituição.

Em sequência, na avaliação da farmacoterapia constatou-se que 5\% das prescrições contêm medicamentos que, segundo critério, deve-se evitar em determinadas condições clínicas/doenças. Na Tabela 1 pode-se definir quais são esses medicamentos e em quais condições devem ser evitados, além do número de prescrições que os mesmos aparecem.

Tabela 1 - Medicamentos prescritos em condições clínicas que devem ser evitadas

\begin{tabular}{|c|c|c|}
\hline Medicamentos & Condições a evitar & Total de Prescrições \\
\hline Bupropiona & Convulsões & 1 \\
\hline Topiramato & Histórico de Quedas e Fraturas & 1 \\
\hline Ácido Valpróico & Histórico de Quedas e Fraturas & 1 \\
Donepezila & Histórico de Síncope & 1 \\
Carvedilol & Doença Pulmonar Obstrutiva & 5 \\
\hline Clopidogrel & Distúrbios Hemorrágicos & 1 \\
Galantamina & Histórico de Síncope & 1 \\
\hline Pregabalina & Histórico de Quedas e Fraturas & 1 \\
\hline
\end{tabular}

Dando seguimento as avaliações, constatou-se que dentre os 217 medicamentos prescritos, $8 \%$ devem ser utilizados com cautela em idosos, dentre estes pode-se considerar a preeminência do uso de Ácido acetilsalicílico (AAS), estando presente em $61 \%$ das prescrições que continham medicamentos inapropriados ou que devem ser utilizados com cautela, seguido da Carbamazepina (17\%), Cilostazol (11\%), Lamotrigina $(6 \%)$ e por último a Dabigatrana $(5 \%)$.

O último parâmetro analisado trata-se das interações medicamentosas de maior relevância clínica, encontradas durante a análise do tratamento farmacológico.

Foram encontradas 216 interações medicamentosas, sendo 91\% (196) interações moderadas e $9 \%$ (20) interações graves. Dentre as interações graves podemos pontuar na Tabela 2 os principais efeitos adversos que o paciente poderá apresentar como resultado do uso concomitante desses fármacos. 
Tabela 2 - Efeitos adversos resultantes das interações medicamentosas graves

\begin{tabular}{|c|c|c|}
\hline Interação Medicamentosa & Efeitos Adversos & Prescrições \\
\hline Risperidona $\times$ Citalopram & Risco de Arritmia & 1 \\
\hline $\begin{array}{c}\text { Fenitoína x Fenobarbital x } \\
\text { Nimodipino }\end{array}$ & $\begin{array}{c}\text { Diminuição da Concentração } \\
\text { de Nimodipino }\end{array}$ & 1 \\
\hline Omeprazol x Cilostazol & $\begin{array}{c}\text { Aumento da Concentração } \\
\text { de Cilostazol }\end{array}$ & 1 \\
\hline Anlodipino $\times$ Sinvastatina & $\begin{array}{c}\text { Aumento da Concentração } \\
\text { de Sinvastatina }\end{array}$ & 1 \\
\hline Bupropiona x Fluoxetina & $\begin{array}{l}\text { Aumento do Risco de } \\
\text { Convulsão }\end{array}$ & 1 \\
\hline $\begin{array}{c}\text { Topiramato x Risperidona x } \\
\text { Quetiapina }\end{array}$ & $\begin{array}{c}\text { Aumento da Temperatura } \\
\text { Corporal }\end{array}$ & 1 \\
\hline Amitriptilina $x$ Fluoxetina & $\begin{array}{l}\text { Risco de Sedação, } \\
\text { Constipação e Retenção } \\
\text { Urinária }\end{array}$ & 2 \\
\hline $\begin{array}{c}\text { Amiodarona x Furosemida x } \\
\text { Quetiapina }\end{array}$ & Risco de Arritmia & 1 \\
\hline Amiodarona $\times$ Sinvastatina & $\begin{array}{c}\text { Aumento da Concentração } \\
\text { de Sinvastatina }\end{array}$ & 1 \\
\hline Haloperidol x Clorpromazina & Risco de Arritmia & 1 \\
\hline Ergocalciferol x Colecalciferol & Risco de Toxicidade & 1 \\
\hline Haloperidol x Citalopram & Risco de Arritmia & 1 \\
\hline Omeprazol x Citalopram & $\begin{array}{c}\text { Aumento da Concentração } \\
\text { de Citalopram }\end{array}$ & 1 \\
\hline Enalapril x Espironolactona & Risco de Hipercalemia & 1 \\
\hline $\begin{array}{l}\text { Hidroclorotiazida } x \\
\text { Espironolactona }\end{array}$ & Risco de Hipercalemia & 1 \\
\hline Quetiapina x Escitalopram & Risco de Arritmia & 1 \\
\hline
\end{tabular}

\section{Discussão}

O presente estudo demonstrou que $60 \%$ dos pacientes institucionalizados eram do sexo feminino, semelhantemente a resultados obtidos em outros estudos (10-12), esse dado justifica-se pelo aumento da longevidade feminina em paralelo com a diminuição da taxa de mortalidade (10). Prevalece na amostra a faixa etária de 71 a 85 anos, resultado este conveniente em comparação ao perfil de idosos residentes em instituições de longa permanência (13).

Dos 35 idosos estudados, $43 \%$ possuem de 3 a 4 comorbidades e $6 \%$ possuem mais de 5 comorbidades, destacando-se a Hipertensão Arterial Sistêmica com 23\% de portadores, seguida da Doença de Alzheimer 8\%, Diabetes 7\%, Cardiopatias 7\% e sequelas de AVC $7 \%$. Trata-se de um perfil comum entre pacientes com mais de 60 anos quando se contrasta os dados obtidos com estudos pré-existentes (11-14). Em contrapartida, podese observar um número acentuado de idosos em tratamento polimedicamentosos, $66 \%$ estão fazendo uso concomitante de 05 a 10 medicamentos e $8 \%$ fazem uso de mais de 10 medicamentos.

Esse ponto apresenta uma variável preocupante devido as alterações fisiológicas características do envelhecimento, responsáveis essas pelo aumento da vulnerabilidade do paciente idoso aos fármacos administrados (15). Coincidente ao exposto, pode-se pontuar as transformações na farmacocinética, relacionadas a atenuação do funcionamento dos órgãos, primordialmente quando se faz alusão a medicamentos com eliminação via renal ou com mecanismo de primeira passagem hepático, além das 
mudanças no perfil de absorção com a diminuição da mobilidade gastrointestinal e elevação do pH gástrico (16).

É característico o declínio de massa muscular, paralelo ao aumento de massa gorda com o avanço da idade, bem como a perda do volume hídrico corporal, resultando na redução da distribuição de medicamentos com característica hidrofílica e acúmulo de fármacos lipofílicos no organismo, como por exemplo os benzodiazepínicos. Os medicamentos estimulantes também perdem uma parcela de sua eficácia devido ao declínio de massa cerebral e do fluxo sanguíneo, favorecendo os efeitos de fármacos antidepressivos, sedativos e antipsicóticos. Sobre outra perspectiva, podemos pontuar as alterações cardiovasculares principalmente com a perda de elasticidade, responsável pelo aumento da pressão arterial e baixo reflexo barorreceptor, originando comumente episódios de hipotensão postural (16-18).

Alterações também na farmacodinâmica corroboram com a intensificação da suscetibilidade ao medicamento, devido a variação na interação entre o fármaco $\mathrm{x}$ receptor e mudanças no mecanismo homeostático, ocorrendo especialmente em medicamentos que agem alterando as funções cognitivas. Um exemplo muito citado são as reações adversas causadas por fármacos com ação anticolinérgica, os idosos são muito propensos a sonolência, confusão, constipação, retenção urinária, hipotensão, entre outras reações, quando em uso de medicamentos dessa classe. Essas modificações fisiológicas criam um cenário de alto risco de efeitos adversos, interações medicamentosas e toxicidade (15-19).

Seguindo a avaliação farmacoterapêutica da amostra conforme o critério de Beers (20) publicado recentemente em 2019, agregado aos conceitos de START/STOPP (21) publicado em 2010, classificou-se os medicamentos em três eixos de análise, sendo o primeiro eixo composto por medicamentos potencialmente inapropriados para idosos, que atingiu o total de $30 \%$ dos fármacos prescritos, com prevalência de medicamentos atuantes no SNC, como por exemplo, os antipsicóticos atípicos, benzodiazepínicos e inibidores seletivos da recaptação de serotonina. Em geral, os idosos possuem maior vulnerabilidade a essas classes farmacológicas devido a reduções no seu metabolismo, conforme exposto anteriormente, agregando um risco eminente de complicações cognitivas, delirium, quedas, fraturas, comprometimento da função psicomotora, exacerbação de disfunção respiratória crônica ou aguda além de hipotensão ortostática (9, 20-22).

O segundo eixo avaliado trata-se de medicamentos potencialmente inapropriados em determinadas condições clínicas/doenças, este aparece como 5\% do total de medicamentos prescritos, desta vez trazendo um alerta referente ao uso do Carvedilol em pacientes com Doença Pulmonar Obstrutiva Crônica, devido ao risco maior de broncoespasmo (9), em paralelo ao uso de medicamentos com ação no SNC em pacientes com histórico de quedas e fraturas $(20,21)$.

O terceiro eixo apresentado traz referência ao uso de medicamentos potencialmente inapropriados que devem ser utilizados com cautela em idosos, estes representam $8 \%$ do total de medicamentos utilizados, com predomínio de $61 \%$ de prescrições contendo Ácido Acetil Salicílico. Segundo critério de Beers, o risco de sangramento com o uso prolongado de AAS aumenta acentuadamente em pacientes com idade mais avançada e o uso deve ser monitorado (20).

Devido aos dados amostrais apresentarem indivíduos polimedicados, aspecto este que aparece em concordância com outros estudos $(10,23,24)$, realizou-se a quantificação de interações medicamentosas presentes nas prescrições em análise, através do auxílio ferramentas online (25-27).

Categorizou-se 216 interações medicamentosas, presentes em 30 prescrições e 
catalogadas em $91 \%$ de interações moderadas e $9 \%$ de interações graves, bem como, apresentou-se os principais efeitos adversos resultantes dessas interações graves, em destaque o risco de desenvolvimento de Arritmia cardíaca, além do aumento da concentração de determinados fármacos no sangue (25-27). Trata-se de um fator alarmante que atesta a necessidade de reavaliação periódica da farmacoterapia dos pacientes idosos (15).

Grande parte das interações medicamentosas e uso de medicamentos potencialmente inapropriados, trata-se de uma consequência da prática polimedicamentosa, estudos realizados na Irlanda apontam que a probabilidade de um paciente que possui uma terapia com mais de cinco medicamentos estar em uso de um medicamento inadequado é 3,3 vezes maior do que um paciente que faz uso de uma quantidade menor de fármacos (28). Outro estudo realizado nos Estados Unidos apontou que $33 \%$ dos pacientes desenvolveram efeitos adversos a medicamentos e o fator mais fortemente associado foi o elevado número de medicamentos utilizados simultaneamente (29).

Na Noruega foi levantado um estudo de quantificação de interações medicamentosas, no qual $48 \%$ dos pacientes residentes em instituições de longa permanência possuíam interações em sua farmacoterapia, e mais uma vez esse número foi significativamente correlacionado a polifarmácia (30).

Apesar de os dados apresentados nos estudos realizados na Irlanda (28) e nos Estados Unidos (29) não possuírem relação com idade ou sexo do paciente, sabe-se que as interações medicamentosas adjacentes aos fatores fisiológicos causam reações adversas de alto risco ao idoso. Um estudo apontou que o risco de hospitalização por reações é sete a quatro vezes maior em um idoso do que em um paciente de idade jovem (24).

Nos Estados Unidos 5\% a 10\% das internações estão relacionadas a interações e toxicidades medicamentosas, isso acaba se tornando além de um problema grave de saúde, um problema financeiro, pois estima-se que a cada 1 dólar gasto com medicamentos e investimentos hospitalares, 1,33 dólares são necessários para tratar casos de morbimortalidade por medicamentos (31). No Brasil não foram encontrados estudos que quantifiquem a repercussão financeira nos sistemas de saúde.

As reações a medicamentos são corriqueiras causas de doenças iatrogênicas, pois causam reações que podem ser facilmente confundidas com o aparecimento de novas patologias geriátricas, possivelmente levando o prescritor a adicionar um novo medicamento na terapia, criando uma cascata de prescrições (32).

Uma maneira de prevenir a doença iatrogênica e a cascata de prescrições é considerar que os sinais e sintomas apresentados possam estar diretamente correlacionados a farmacoterapia, como um efeito adverso, interações medicamentosas, aumento da concentração e redução do metabolismo ou eliminação do medicamento devido alterações fisiológicas (33).

O uso de medicamentos contínuos por idosos precisa ser reavaliado frequentemente pelos profissionais de saúde e principalmente pelo prescritor durante as consultas de rotina, com o intuito de evitar ao máximo reações adversas e possíveis doenças iatrogênicas, a fim de reduzir internações hospitalares e garantir ao paciente idoso segurança, eficácia e qualidade de vida durante o tratamento.

Por se tratar de uma instituição de longa permanência de caráter filantrópico o acesso do paciente a um único prescritor durante o período de tratamento é algo complicado de conseguir, ou seja, a avaliação detalhada, individual e o monitoramento contínuo do tratamento é um verdadeiro desafio atualmente. Devido a isso a conscientização dos profissionais é de extrema importância para que o paciente não seja tratado em fragmentos, mas sim como um todo, em conjunto. 


\section{Conclusões}

O desenvolvimento do referido estudo possibilitou demonstrar o uso exacerbado de medicamentos inapropriados para idosos e destacar como consequência o elevado número de interações medicamentosas, bem como a gravidade dos efeitos adversos que podem ser ocasionados pelo uso concomitante destes fármacos.

A elevada suscetibilidade do paciente idoso devido as mudanças fisiológicas características do envelhecimento, frente a farmacocinética e farmacodinâmica dos medicamentos acarretam muitas vezes em problemas maiores do que aqueles que originaram a necessidade do tratamento farmacológico.

É papel do farmacêutico em suas atribuições clínicas contribuir com o uso racional de medicamentos, bem como conscientizar os demais profissionais de saúde sobre a importância do cuidado com a saúde do idoso, além da implementação de ferramentas necessárias para a definição da melhor terapia destinada a cada paciente em sua individualidade, garantindo o acesso a medicamentos seguros e adequados ao tratamento.

Por fim, cabe aos profissionais de saúde desenvolver ações voltadas ao paciente idoso, reforçando a importância da autonomia do mesmo em relação ao tratamento, alertando sobre os riscos da automedicação e principalmente sobre os efeitos adversos que podem aparecer e quais medidas devem ser tomadas nessas situações.

\section{Referências}

1. Brasil. Lei $\mathrm{n}^{\mathrm{o}} 10.741$ de $1^{\circ}$ de outubro de 2003.Dispõe sobre o Estatuto do Idoso e dá outras providências. Diário Oficial da União. 1 out 2003.

2. Closs V, Schwanke C. A evolução do índice de envelhecimento no Brasil, nas suas regiões e unidades federativas no período de 1970 a 2010. Revista Brasileira de Geriatria e Gerontologia. 2012; 15(3): 443-458.

3. Alves J. Transição demográfica, transição da estrutura etária e envelhecimento. Revista Portal de Divulgação. 2014; 40(15): 8-15.

4. World Health Organization. Envelhecimento ativo: uma política de saúde / World Health Organization; tradução Suzana Gontijo. Brasília: Organização Pan-Americana da Saúde, 2005.

5 Brasil. Ministério da Saúde. Secretaria de Ciência, Tecnologia e Insumos Estratégicos. Departamento de Assistência Farmacêutica e Insumos Estratégicos. Capacitação para implantação dos serviços de clínica farmacêutica / Ministério da Saúde, Secretaria de Ciência, Tecnologia e Insumos Estratégicos. Departamento de Assistência Farmacêutica e Insumos Estratégicos. Brasília: Ministério da Saúde, 2014.

6. Souza TT. Morbidade e mortalidade relacionadas a medicamentos: revisão sistemática e meta-análise de estudos observacionais [Dissertação de mestrado]. Curitiba: Pós-graduação em Ciências Farmacêuticas, Universidade Federal do Paraná; 2013.

7. Page RL, Linnebur SA, Bryant LL, Ruscin JM. Inappropriate prescribing in the hospitalized elderly patient: defining the problem, evaluation tools, and possible solutions. Clinical Interventions in Aging. 2010; 5: 75-87.

8. Oliveira MG, Amorim WW, Oliveira CRB, Coqueiro HL, Gusmão LC, Passos LC. Consenso Brasileiro de medicamentos potencialmente inapropriados para idosos. Sociedade Brasileira de Geriatria e Gerontologia. Informativos. Bahia. 2016.

9. Tiago JPT. Polimedicação No Idoso: Artigo de Revisão [Tese de mestrado]. Coimbra: Faculdade de Medicina da Universidade De Coimbra, 2014. 68 p.

10. Rosa ASKC, Costa BP, Kapper CP, Dalmas GCS, Sbroglio LL, Andreis L, Lampert MA. Identificação de prescrição inapropriada em ambulatório de Geriatria utilizando os Critérios Stopp e Start. Revista Brasileira de Geriatria e Gerontologia. 2016; 19(5): 871-878.

11. Lopes LM, Figueiredo TP, Costa SC, Reis AMM. Utilização de medicamentos potencialmente inapropriados por idosos em domić́lio. Revista Ciência e Saúde Coletiva. 2016; 21(11): 3429-3438.

12. Coelho Filho JM, Marcopito LF, Castelo A. Perfil de utilização de medicamentos por idosos em área urbana no Nordeste do Brasil. Revista Saúde Pública. 2004; 38(4): 557-564.

13. Freitas MC, Guedes MVC, Galiza FT, Nogueira JM, Onofre, MR. Idosos residentes em uma instituição de longa permanência: adaptação à luz de Callista Roy. Revista Brasileira de Enfermagem. 2014; 67 (6): 905-912. 
14. Vaz CSSB. Medicamentos Potencialmente Inapropriados para Idosos (A realidade de um serviço de medicina) [Dissertação de Mestrado]. Coimbra: Universidade de Coimbra, 2012.

15. Gorzoni ML, Fabri RMA, Pires SL. Medicamentos Potencialmente Inapropriados para Idosos. Revista da Associação Médica Brasileira. 2012; 58(4): 442-446.

16. Galvão C. O idoso polimedicado - estratégias para melhorar a prescrição. Revista Portuguesa Clínica Geral. 2006; 22: 747-752.

17. Rezende JAI, Girotto E. Risco de polimedicação em idosos: Uma revisão. Revista Uningá, 2019; 56(1): 66-76.

18. Oliveira AM. Fatores de risco associados a polifarmacia no idoso [Trabalho de Conclusão de Curso] Campos Gerais: Universidade Federal de Minas Gerais; 2013.

19. Ruscin JM, Linnebur SA. Manual MSD [homepage da internet]. Farmacodinâmica em idosos [acesso 25 de outubro de 2019]. Disponível em: https://www.msdmanuals.com/ptbr/profissional/geriatria/terapia-medicamentosa-em-idosos/farmacodinâmica-emidosos\#v1132595_pt.

20. American Geriatrics Society Beers Criteria ${ }^{\circledR}$ Update Expert Panel. American Geriatrics Society 2019 Updated AGS Beers Criteria ${ }^{\circledR}$ for Potentially Inappropriate Medication Use in Older Adults. Journal of the American Geriatric Society. 2019; 67(4): 674-694.

21. Mahony DO, Gallagher P, Ryan C, Byrne S, Connor MO, Kennedy J. Stopp e start criteria: A new approach to detecting potentially inappropriate prescribing in old age. European Geriatric Medicine. 2010; 1(1): 45-51.

22. Instituto para Práticas Seguras no Uso de Medicamentos. Medicamentos Potencialmente Inadequados para Idosos.2017 [acesso 04 de outubro de 2019]. Disponível em: https://www.ismp-brasil.org/site/wpcontent/uploads/2017/09/is 0006 17a boletim agosto ismp 210x276mm_v2.pdf.

23. Araujo CMC, Magalhães SMS, Chaimowicz F. Uso de Medicamentos Inadequados e Polifarmácia entre Idosos do Programa Saúde da Família. Latin American Journal of Pharmacy. 2010; 29(2): 178184.

24. Secoli, SR. Polifarmácia: interações e reações adversas no uso de medicamentos por idosos. Revista Brasileira de Enfermagem. 2010; 63(1): 136-140.

25. UptoDate [homepage na internet]. Interactions Checker [acesso 10 de outubro de 2019]. Disponível em: https://www.uptodate.com/home.

26. Drugs [homepage na internet]. Interactions Checker [acesso 10 de outubro de 2019]. Disponível em: https://www.drugs.com.

27. Topol, EJ. Medscape [homepage na internet]. Interactions Checker [acesso 10 de outubro de 2019]. Disponível em: https://www.medscape.com.

28. Gallagher PF, O’Connor MN, O’Mahony D. Prevention of potentially inappropriate prescribing for elderly patients: a randomized controlled trial using STOPP/ START criteria. Clin Pharmacol Ther. 2011; 14(2): 283-290.

29. Steinman MA, Lund BC, Miao Y, Boscardin WJ, Kaboli PJ. Geriatric conditions, medication use, and risk of adverse drug events in a predominantly male, older veteran population. Am Geriatr Soc. 2011; 59(4): 615-621.

30. Halvorsen KH, Granas AG, Engeland A, Ruths S. Prescribing quality for older people in Norwegian nursing homes and home nursing services using multidose dispensed drugs. Pharmacoepidemiol Drug Saf. 2012; 21(9): 929-936.

31. McLean AJ, Le Couteur DG. Aging biology and geriatric clinical pharmacology. Pharmacol Ver. 2004; 56(2): 163-84.

32. Cinthia M, Willians C. Using Medications Appropriately in Older Adults. Rev American Family Physician. 2002; 66(10): 1917-1924.

33. Chaimowicz F. Saúde do Idoso. 2. ed. Belo Horizonte: Nescon UFMG, 2013. 